\title{
CP violation results on Kaons by the NA48 experiment
}

\author{
M. Lenti ${ }^{\mathrm{a} *}$ \\ ${ }^{a}$ INFN Sezione di Firenze, \\ Via G.Sansone 1, 50019 Sesto F.(Firenze), Italy
}

Recent results on CP violation on kaons by the CERN NA48 experiment will be discussed. A preliminary measurement of the charge asymmetry in $K_{L} \rightarrow \pi^{ \pm} e^{\mp} \nu$ has been made: $\delta_{L}^{\epsilon}=\left(3.317 \pm 0.070_{\text {stat }} \pm 0.072_{\text {syst }}\right) \times 10^{-3}$. A search for $K_{S} \rightarrow 3 \pi^{0}$ decays has been performed with a preliminary estimation of the real and imaginary part of the $\eta_{000}$ parameter: $\operatorname{Re}\left(\eta_{000}\right)=\left(-2.6 \pm 1.0_{\text {stat }} \pm 0.5_{\text {syst }}\right) \times 10^{-2}$ and $\operatorname{Im}\left(\eta_{000}\right)=\left(-3.4 \pm 1.0_{\text {stat }} \pm 1.1_{\text {syst }}\right) \times 10^{-2}$. The angular asymmetry in $K_{L} \rightarrow \pi^{+} \pi^{-} e^{+} e^{-}$has been measured to be $A_{\phi}^{L}=\left(14.2 \pm 3.0_{\text {stat }} \pm 1.9_{\text {syst }}\right) \%$, while no asymmetry has been observed in $K_{S} \rightarrow \pi^{+} \pi^{-} e^{+} e^{-}: A_{\phi}^{S}=\left(0.5 \pm 4.0_{\text {stat }} \pm 1.6_{\text {syst }}\right) \%$. Finally, the direct $\mathrm{CP}$ violation in $K_{S, L} \rightarrow 2 \pi$ has been clearly settled, with $\operatorname{Re}(\epsilon / \epsilon)=(14.7 \pm 2.2) \times 10^{-4}$.

\section{INTRODUCTION}

Neutral Kaons were the place where CP violation was discovered for the first time. NA48 is an experiment dedicated to the precise study of $\mathrm{CP}$ violation in the neutral Kaon system: its beam setup is made of two neutral beams [1], one dominated by $K_{L}$ decays, the other by $K_{S}$ decays.

\subsection{The beam setup}

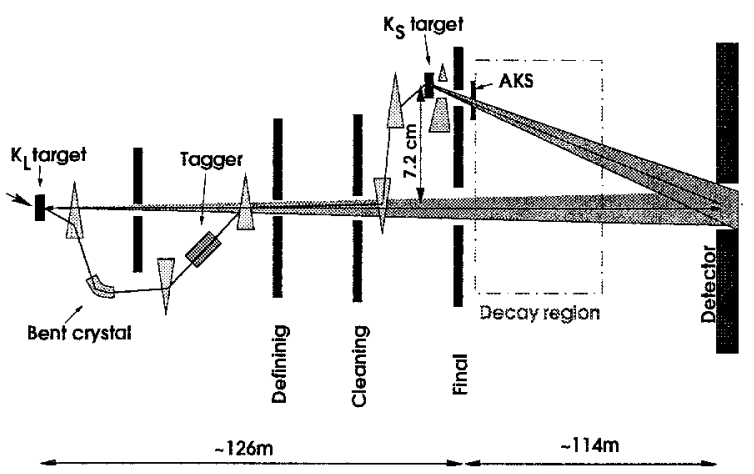

Figure 1. The NA48 beam setup.

A primary proton beam from the CERN Su- per Proton Syncroton impinges, with a nominal flux of $1.5 \times 10^{12}$ particles per spill, on a beryllium target ("far target") $40 \mathrm{~cm}$ long and $2 \mathrm{~mm}$ wide; after a magnet sweeping sector and several stages of collimation, a neutral beam is formed. The exit face of the last collimator is located $\mathbf{1 2 6}$ $m$ downstream of the target, at the entrance of the fiducial kaon decay region.

The primary protons which have not interacted in the target are deflected towards a bent silicon crystal: a small fraction of these protons is channeled by the crystal and form a secondary proton beam which is transported towards a second beryllium target ("near target"); after only $6 \mathrm{~m}$ of magnet sweeping and collimation another neutral beam is formed.

The two neutral beams can be present at the same time ("simultaneous beam runs") or one per time ("far target run" or "near target run") if only $K_{L}$ or $K_{S}$ decays have to be studied.

The secondary proton beam is usually at much lower intensity (about $3 \times 10^{7}$ particles per spill) with respect to the primary one. In some special runs ("High Intensity $K_{S}$ runs") the primary proton beam is sent directly to the second target, removing the first target and by-passing the silicon crystal; the proton beam is attenuated and collimated to the desired intensity far upstream of the second target.

*on behalf of the NA48 collaboration 


\subsection{The Detectors}

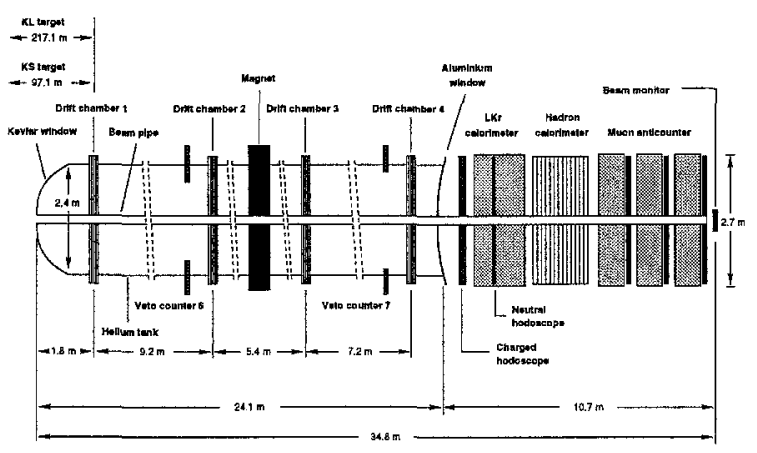

Figure 2. The NA48 detectors.

The detectors are located downstream of the kaon decay volume which lies inside a large, $90 \mathrm{~m}$ long, vacuum tank terminated by a $0.3 \%$ radiation lenghts thick Kevlar window; starting at the center of the Kevlar window, a $16 \mathrm{~cm}$ diameter vacuum beam pipe traverses all the detectors to let the neutral beam pass through vacuum.

Charged particles are detected using a high resolution magnetic spectrometer which consists of a dipole magnet with a horizontal transverse momentum kick of $265 \mathrm{MeV} / \mathrm{c}$ and a set of four drift chambers, two of them located upstream of the magnet and two downstream. The magnetic spectrometer is contained inside a tank filled with helium in order to reduce multiple scattering. The momentum resolution is:

$\frac{\sigma_{p}}{p}(\%)=0.48 \oplus 0.009 p(\mathrm{p}$ in $\mathrm{GeV} / \mathrm{c})$

A quasi-homogeneous liquid krypton (LKr) calorimeter is located downstream of the spectrometer. This detector has a $127 \mathrm{~cm}$ long projective tower structure which is made of copperberyllium ribbons extending between the front and the back of the calorimeter with a $\pm 48 \mathrm{mrad}$ accordion geometry. The 13212 readout cells each have a cross-section of $2 \times 2 \mathrm{~cm}^{2}$. The energy resolution is:

$\frac{\sigma_{E}}{E}(\%)=\frac{3.2}{\sqrt{E}} \oplus \frac{9.0}{E} \oplus 0.42(\mathrm{E}$ in $\mathrm{GeV})$
Two planes of scintillators, segmented in horizontal and vertical slabs, form the charged hodoscope, located in between the magnetic spectrometer and the LKr calorimeter; it is used for triggering and measuring the time of charged particles.

Behind the LKr electromagnetic calorimeter, a 6.7 nuclear interaction lenght thick hadronic calorimeter is located, followed by a set of three planes of muon veto counters.

\subsection{Data samples}

Section 2 is based on data collected with the "simultaneous beams" setup in the year 1999 .

Section 3 is based on data collected in the year 2000 with a 40 days long "near target high intensity run" and a 40 days long "far target run". The magnetic spectrometer was not present in these two runs.

Section 4 is based on data collected with the "simultaneous beams" setup in the year 1998 and 1999 and with the "near target" setup in a 2 days long high intensity run in 1999.

Section 5 is based on data collected with the "simultaneous beams" setup in the year 1997, 1998, 1999 and 2001.

\section{CHARGE ASYMMETRY IN $K_{L} \rightarrow$ $\pi^{ \pm} e^{\mp} \nu$}

The charge asymmetry in $K_{e 3}$ decays is defined as

$\delta_{L}^{e} \equiv \frac{\Gamma\left(K_{L} \rightarrow \pi^{-} e^{+} \nu_{e}\right)-\Gamma\left(K_{L} \rightarrow \pi^{+} e^{-} \bar{\nu}_{e}\right)}{\Gamma\left(K_{L} \rightarrow \pi^{-} e^{+} \nu_{e}\right)+\Gamma\left(K_{L} \rightarrow \pi^{+} e^{-} \bar{\nu}_{e}\right)}$

If CPT symmetry holds, this observable is equal to $2 \operatorname{Re}(\epsilon)$.

During the 2001 run about $2 \times 10^{8} K_{e 3}$ decays have been collected, along with $\pi \pi$ samples for $\operatorname{Re}(\epsilon / / \epsilon)$ measurement. The systematic effects due to detector asymmetry are reduced by regular changes of the spectrometer magnet polarity. The backgrounds could be reduced to a negligible level by selection cuts. The main source of systematic uncertainties arises from charge dependent interactions of $\pi^{ \pm}$in the electromagnetic calorimeter which are reflected in asymmetric trigger and particle identification efficiency (Table 1). 
Table 1: Systematic corrections to $\delta_{L}^{e}$

\begin{tabular}{ll}
\hline & in $10^{-5}$ \\
\hline Trigger & $+26.2 \pm 6.0$ \\
Punch trough & $-1.4 \pm 3.5$ \\
Pion ID & $-\mathbf{1 7 . 1} \pm 2.4$ \\
Acceptance & \pm 0.5 \\
Background & \pm 0.5 \\
\hline
\end{tabular}

The corrections are calculated using various control samples involving charged pions, like $K_{S} \rightarrow \pi^{+} \pi^{-}$and $K_{L} \rightarrow \pi^{+} \pi^{-} \pi^{0}$.

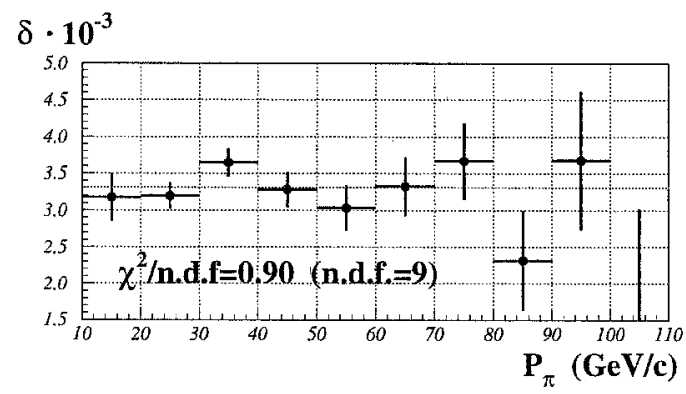

Figure 3. $\delta_{L}^{e}$ as a function of pion momentum.

The charge asymmetry in $K_{e 3}$ decays, after all the corrections, is shown in Fig. 3 in bins of pion momentum.

The preliminary result is

$\delta_{L}^{e}=\left(3.317 \pm 0.070_{\text {stat }} \pm 0.072_{\text {syst }}\right) \times 10^{-3}$

which is in good agreement with previous measurements [2].

\section{SEARCH FOR $K_{S} \rightarrow 3 \pi^{0}$}

The CP violating parameter $\eta_{000}$ is defined as

$\eta_{000} \equiv \frac{A\left(K_{S} \rightarrow \pi^{0} \pi^{0} \pi^{0}\right)}{A\left(K_{L} \rightarrow \pi^{0} \pi^{0} \pi^{0}\right)}$

If CPT symmetry is conserved, the real part of $\eta_{000}$ is given by CP violation in mixing and is equal to $\operatorname{Re}(\epsilon)$ while the imaginary part can be sensitive to direct $\mathrm{CP}$ violation.
The data for this measurement has been collected during the last period of 2000 data taking in special conditions with high intensity near target beam and without the spectrometer. Almost $6.5 \times 10^{6} K^{0} \rightarrow \pi^{0} \pi^{0} \pi^{0}$ events have been collected. This data is normalized to pure $K_{L} \rightarrow$ $\pi^{0} \pi^{0} \pi^{0}$ sample from far-target beam run (about $155 \times 10^{6}$ events) immediately preceding the neartarget run in practically the same detector conditions, which lead to cancellation of the acceptance in the first order. The result is obtained from a fit to a function

$$
\begin{aligned}
& f(E, t)=\frac{I_{3 \pi^{0}}^{n e a r}}{I_{3 \pi^{0}}^{f a r}}=A(E)\left[1+\left|\eta_{000}\right|^{2} e^{i / \tau_{L}-t / \tau_{S}}\right. \\
& +2 D(E) e^{t / 2 \tau_{L}-t / 2 \tau_{S}}\left(\operatorname{Re}\left(\eta_{000}\right) \cos (\Delta m t)\right. \\
& \left.\left.-I m\left(\eta_{000}\right) \sin (\Delta m t)\right)\right]
\end{aligned}
$$

in kaon energy $E$ and proper time $t$ bins. The $D(E)$ denotes $K^{0}-\bar{K}^{0}$ production asymmetry (dilution) at the target and is fixed to the values measured by NA31 [3].

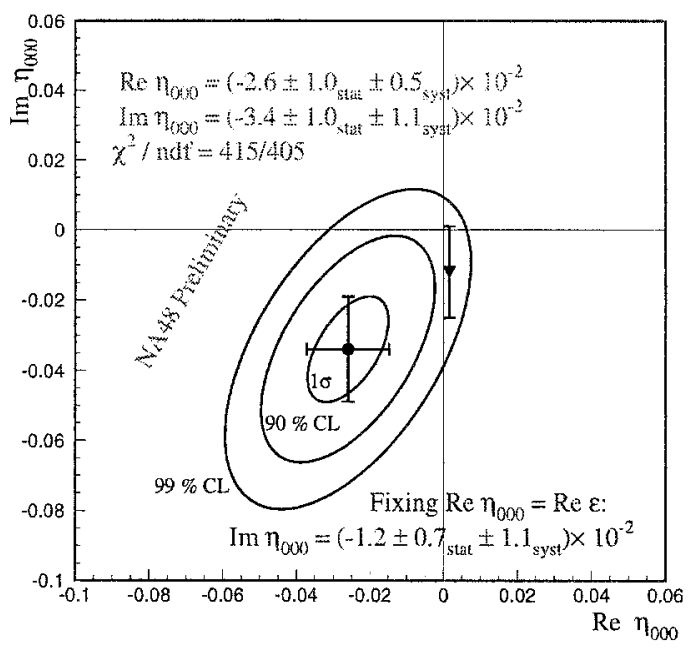

Figure 4. Preliminary result of the $\eta_{000}$ fit. 
The fit results in (Fig.4)

$$
\begin{aligned}
& \operatorname{Re}\left(\eta_{000}\right)=\left(-2.6 \pm 1.0_{\text {stat }} \pm 0.5_{\text {syst }}\right) \times 10^{-2} \\
& \operatorname{Im}\left(\eta_{000}\right)=\left(-3.4 \pm 1.0_{\text {stat }} \pm 1.1_{\text {syst }}\right) \times 10^{-2}
\end{aligned}
$$

The systematic uncertainties are summarised in Table 2.

Table 2: uncertainties in $\eta_{000}$

\begin{tabular}{lll}
\hline & in $10^{-2}$ & \\
& $\operatorname{Re}\left(\eta_{000}\right)$ & $\operatorname{Im}\left(\eta_{000}\right)$ \\
\hline Accidentals & \pm 0.1 & \pm 0.6 \\
Energy scale & \pm 0.1 & \pm 0.1 \\
$K^{0}-\bar{K}^{0}$ dilution & \pm 0.3 & \pm 0.4 \\
Acceptance & \pm 0.3 & \pm 0.8 \\
Binning & \pm 0.1 & \pm 0.2 \\
\hline Total & \pm 0.5 & \pm 1.1
\end{tabular}

The uncertainty of this preliminary measurement is ten times smaller than the best previously published result by CPLEAR [4].

Assuming CPT conservation and fixing the real part of $\eta_{000}$ to $\operatorname{Re}(\epsilon)=1.6 \times 10^{-3}$, the imaginary part of $\eta_{000}$ can be constrained with better precision to

$\operatorname{Im}\left(\eta_{000}\right)=\left(-1.2 \pm 0.7_{\text {stat }} \pm 1.1_{\text {syst }}\right) \times 10^{-2}$

This corresponds to $B R\left(K_{S} \rightarrow \pi^{0} \pi^{0} \pi^{0}\right)<3.0 \times$ $10^{-7}$ at $90 \% \mathrm{CL}$. This result improves by two orders of magnitude the best limit obtained by the SND collaboration [5].

\section{ANGULAR ASYMMETRY IN $K_{L} \rightarrow$ $\pi^{+} \pi^{-} e^{+} e^{-}$}

The $K_{L} \rightarrow \pi^{+} \pi^{-} e^{+} e^{-}$decay is dominated by the CP-violating inner bremsstrahlung (IB) term and by the CP-conserving direct M1 emission (DE) graph. The interference between the IB $(\mathrm{CP}=+1)$ and the DE $(\mathrm{CP}=-1)$ amplitudes produces a CP-violating circular polarization of the $\gamma^{*}$. If $\phi$ is the angle between the $e^{+} e^{-}$and $\pi^{+} \pi^{-}$planes, the following asymmetry can be measured:

$A_{\phi}^{L}=\frac{N_{\sin \phi \cos \phi>0}-N_{\sin \phi \cos \phi<0}}{N_{\sin \phi \cos \phi>0}+N_{\sin \phi \cos \phi<0}}$

The prediction by the Heiliger and Sehgal[6] model is $A_{\phi} \approx 14 \%$.
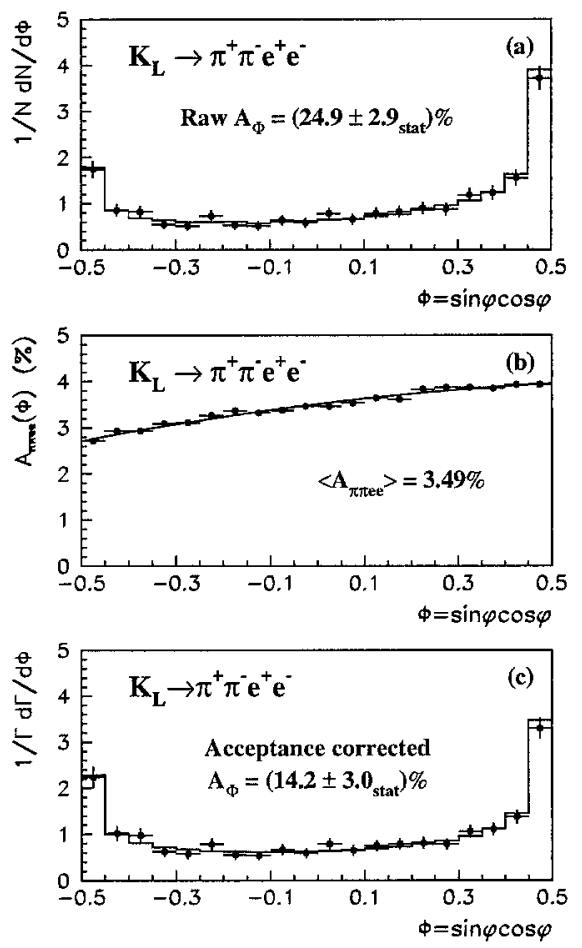

Figure 5. Distribution of $K_{L} \rightarrow \pi^{+} \pi^{-} e^{+} e^{-}$ events in the angular variable $\sin \phi \cos \phi$ before(a) and after(c) acceptance corrections. The histograms are Monte Carlo predictions. Acceptance as a function of $\sin \phi \cos \phi$ (b); the solid line is a polynomial fit to the Monte Carlo calculation.

$1162 K_{L} \rightarrow \pi^{+} \pi^{-} e^{+} e^{-}$candidates have been selected in data collected in 1998 and 1999. The angular asymmetry before any acceptance correction is $\left(24.9 \pm 2.9_{\text {stat }}\right) \%$. The measured $A_{\phi}^{L}$, obtained in a model-dependent way by taking into account the acceptance correction is[7]:

$A_{\phi}^{L}=\left(14.2 \pm 3.0_{\text {stat }} \pm 1.9_{\text {syst }}\right) \%$

and it is a clear signature of indirect $\mathrm{CP}$ violation.

The $K_{S} \rightarrow \pi^{+} \pi^{-} e^{+} e^{-}$decay is dominated by the CP-conserving inner bremsstrahlung (IB) term only and no angular asymmetry is expected: 

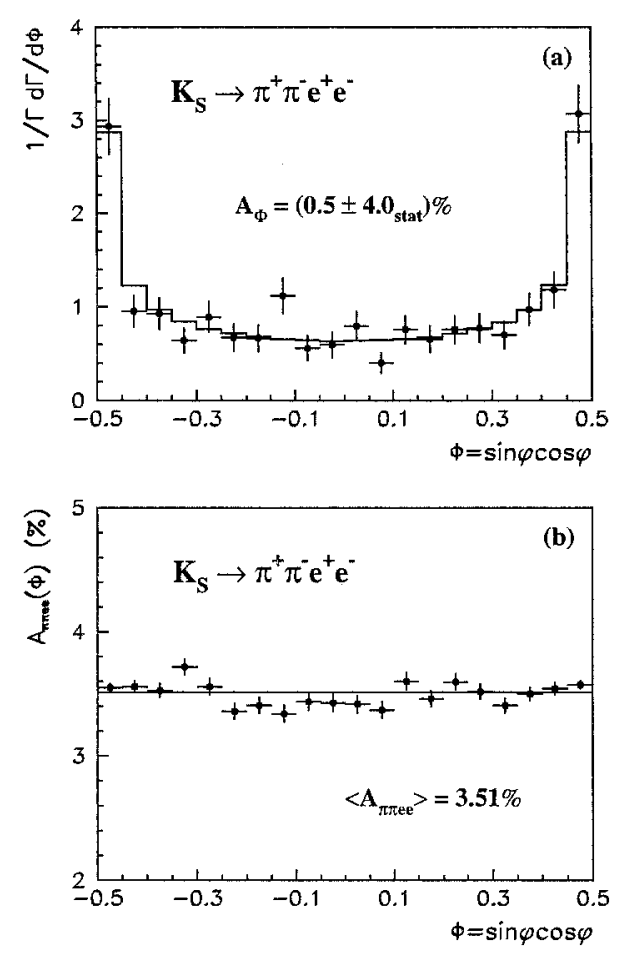

Figure 6. Distribution of events(a) and acceptance(b) for $K_{S} \rightarrow \pi^{+} \pi^{-} e^{+} e^{-}$decays as a function of $\sin \phi \cos \phi$. The histogram in (a) is the Monte Carlo prediction. The solid line in (b) represents the average value of the acceptance.

it is an important cross-check of the $K_{L}$ analysis. Since no interference term is present in the amplitude of this decay mode, the acceptance correction is uniform over the entire $\sin \phi \cos \phi$ domain. Based on the data collected in 1999 the asymmetry is[7]:

$$
A_{\phi}^{S}=\left(0.5 \pm 4.0_{\text {stat }} \pm 1.6_{\text {syst }}\right) \%
$$

which is compatible with a null asymmetry and gives confidence that $A_{\phi}^{L}$ is not generated by detector effects.

\section{DIRECT CP VIOLATION IN $K_{S, L} \rightarrow$ $2 \pi$}

The double ratio of decay widths of $K_{S}$ and $K_{L}$ into 2 pions is given by:

$$
R=\frac{\frac{\Gamma\left(K_{L} \rightarrow \pi^{0} \pi^{0}\right)}{\Gamma\left(K_{S} \rightarrow \pi^{0} \pi^{0}\right)}}{\frac{\Gamma\left(K_{L} \rightarrow \pi^{+} \pi^{-}\right)}{\Gamma\left(K_{S} \rightarrow \pi^{+} \pi^{-}\right)}}=1-6 R e\left(\frac{\epsilon \prime}{\epsilon}\right)
$$

If the four modes are taken in the same decay region and simultaneously, $R$ reduces to the double ratio of the number of decays. The data have been collected in the years 1997, 1998, 1999 and 2001. The main aim of the 2001 run was not only to add more statistics to the already published $R e(\epsilon / / \epsilon)$ result [8] but also to perform additional systematic checks. This was possible due to several changes in the experimental conditions ( $\mathrm{Ta}-$ ble 3 ).

Table 3: Change in conditions of 2001 run

\begin{tabular}{lll}
\hline & $98-99$ & 2001 \\
\hline proton energy & $450 \mathrm{GeV}$ & $400 \mathrm{GeV}$ \\
SPS cycle time & $14.4 \mathrm{~s}$ & $16.8 \mathrm{~s}$ \\
spill lenght & $2.4 \mathrm{~s}$ & $5.2 \mathrm{~s}$ \\
effective s.l. & $1.7 \mathrm{~s}$ & $3.6 \mathrm{~s}$ \\
duty cycle & 0.17 & 0.31 \\
$K_{L}$ beam & & \\
intensity (ppp) & $1.5 \times 10^{12}$ & $2.4 \times 10^{12}$ \\
$K_{S}$ beam & & \\
intensity (ppp) & $3 \times 10^{7}$ & $5 \times 10^{7}$
\end{tabular}

Besides rebuilt drift chambers and change in the beam energy, a significant increase of the duty cycle allowed to collect $29 \%$ of the total statistics with reduced instantaneous beam intensity. The fact that the result is fully compatible with the one obtained from 1997-1999 period confirms the robustness of the $\operatorname{Re}(\epsilon / / \epsilon)$ measurement with respect to the accidental beam activity.

The result from 2001 data only is [9]

$$
\operatorname{Re}(\epsilon / / \epsilon)=(13.7 \pm 3.1) \times 10^{-4}
$$

the result from $97-98-99$ data is [8]

$$
\operatorname{Re}(\epsilon / \epsilon)=(15.3 \pm 2.6) \times 10^{-4}
$$

and the combined result is

$$
\operatorname{Re}(\epsilon / \epsilon)=(14.7 \pm 2.2) \times 10^{-4}
$$




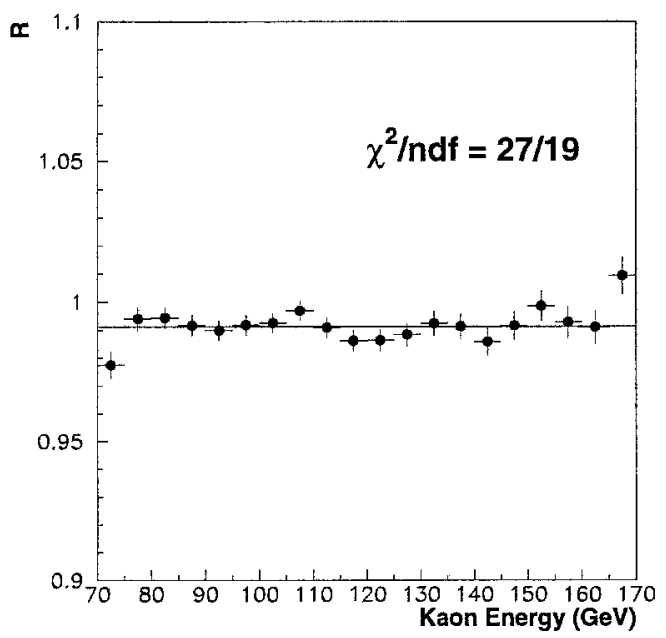

Figure 7. The Double Ratio versus kaon energy.

This final result reaches the design accuracy and leads to a world average of $(16.6 \pm 1.6) \times 10^{-4}$.

\section{CONCLUSIONS}

The NA48 experiment provided several measurement of $\mathrm{CP}$ violation effects in the neutral kaon system. In the year 2003 a search for CP violation in the charged kaons sector will be performed.

\section{REFERENCES}

1. N.Doble et al., Nucl. Instr. Meth. B 119 (1996) 181.

2. A.Alavi-Harati et al., Phys. Rev. Lett. 88 (2002) 181601.

3. G.Barr et al., Phys. Lett. B 317 (1993) 233.

4. A.Angelopoulos et al., Phys. Lett. B 425 (1998) 391.

5. M.N.Achasov et al., Phys. Lett. B 459 (1999) 674.

6. P.Heiliger and L.M.Sehgal, Phys. Rev. D 48 (1993) 4146;

Erratum: Phys. Rev. D 60 (1999) 079902.
7. A.Lai et al., CERN-EP/2003-006, submitted to EPJ.

8. A.Lai et al., Eur. Phys. J. C 22 (2001) 231.

9. R.Batley et al., Phys. Lett. B 544 (2002) 97. 